\title{
XXII.
}

\section{Ueber Ammoniakausscheidnng.}

\section{Vorläuffige Mittheilnng und Erwiderung an Herrn Hallervorden.}

\author{
Von Th. Rumpf in Hamburg.
}

Hallervorden hat in dem 3. Heft des 143. Bandes dieses Archivs eine Arbeit von mir äber Ammoniakausscheidung zum Gegenstand einer Erörterung gemacht und dabei einige Irrthümer als der Aufklärung bedürftig bezeichnet.

Er geht dabei von dem Gedanken aus, dass ich die Sãuren, an welche das $\mathrm{NH}_{3}$ gebunden ist, völlig ausser Acht gelassen babe und citirt demgegenüber eigene frühere Resultate, durch welche er die Frage der $\mathrm{NH}_{3^{-}}$ Ausscheidung für ziemlich erledigt bält.

Die Ergebnisse von Hallervorden waren mir keineswegs unbekannt, und sind auch grösstentheils von wir citirt worden. Icb halte aber Ballervorden's Auffassung in dem allgemeinen Sinne, dass das $\mathrm{NH}_{3}$ die Function der Säureneutralisation im Körper zu üben hat, für falseh.

In meiner ersten, in diesem Archiv erschienenen Arbeit habe ich mich absichtlich recht zurückhaltend bezüglich der gleichzeitigen Säureausscheidung ausgedrückt. Der Grund lag keineswegs in den fehlenden Säurebestimmungen, sondern in der Thatsache, dass meine Resultate sich mit den Anschaungen der früheren Autoren nicht deckten.

Was mir bei meinen mehrjăbrigen Untersuchungen vor Allem auffiel, war der Umstand, dass die Ausscbeidung des $\mathrm{NH}_{3}$ und die der Schwefelsänre und Phosphorsäure nicht parallel ging; an dem einen Tage erreichte die Ausscheidung beider Säuren hăufig den Höhepunkt, während an demselben Tage wenig $\mathrm{NH}_{3}$ ausgeschieden wurde; an einem anderen Tage war die $\mathrm{NH}_{3}$-Ausscheidung sehr boch und diejenige der Phosphorsäure und Schwefelsãure sehr gering. Auch die Chlorausscheidung kann z. B. für die Pneumonie kaum in Betracht kommen.

Diese Befunde mussten den Gedanken nahe legen, dass die Verhältnisse viel complicirter sind, als Hallervorden annahm. - Dass anorganische Säuren zu einer Vermehrung der $\mathrm{NH}_{3}$ - Ausscheidung fübren, wie es Hallervorden zuerst für die Salzsäure nachgewiesen hat, konnte auch nach meinen Versuchen mit Phosphorsäure und Schwefelsāure nicht zweifelhaft sein. Aber auch hier fand sich die überraschende Thatsacbe einer Incon. gruenz der Ausscheidung beider. 
So erhielt ein in Stickstoffgleichgewicht befindlicher 41 jähriger Mann zuerst im Laufe von $3 \frac{1}{2}$ Tagen $1,8112 \cdot \mathrm{P}_{2} \mathrm{O}_{5}$.

Diese erfordern zur Bildung von saurem phosphorsauren Ammonium $0,867 \mathrm{NH}_{3}$. Durch diese Phosphorsäureeinfuhr erfolgte nun eine Mehrausscheidung von Ammoniak, welche in 7 Tagen nur 0,1 im Harn betrug, also der erwarteten Menge nicht entsprach. Aber die gleichzeitige Bestimmung der Phosphorsäure vor und während dieser Zeit ergab, dass in 5 Tagen $2,80=154$ pCt. der Einfuhr wieder ausgeschieden wurden. Bei einem zweiten Versuch, in welchem die gleiche Menge Phosphorsäure in kaum zwei Tagen gegeben wurde, betrug die Vermehrung der $\mathrm{NH}_{3}$ - Ausscheidung $2,42 \mathrm{~g}$, während nur $1,29 \mathrm{P}_{2} \mathrm{O}_{5}=71 \mathrm{pCt}$. der Einfuhr wieder im Harn erschienen. Es ist also hier weit mehr $\mathrm{NH}_{3}$ ausgeschieden, als der eingeführten $\mathrm{P}_{2} \mathrm{O}_{5}$ entsprach, während von letzterer ein beträchtlicher Theil im Körper verblieben ist.

Weiterbin habe ich Einfubr und Ausscheidung verschiedener Ammoniumsalze bei Hunden und Menschen, sowohl mit Bezug auf das Ammoniak, als auf die zugebörige Säure, mit meinem Assistenten Herrn Dr. Kleine einer eingehenden Untersuchung unterworfen. Ueber die erhaltenen Befunde habe ich auf dem Congress für innere Medicin in Wiesbaden Mittheilung gemacht; für die Leser dieses Archivs und Herrn Hollervorden gestatte ich mir einen kurzen Auszug hier mitzutheilen. Es erscheint mir das auch aus dem Grunde zweckmässig zu sein, weil die ausführliche Publication bis zur Erledigung einiger weiteren Fragen noch längere Zeit ausstehen dürfte.

Der erste Versuch wurde an einem 41 jährigen Manne ausgeführt, bei welchem die Bestimmungen des $\mathrm{N}$, des $\mathrm{NH}_{3}$, des $\mathrm{P}_{2} \mathrm{O}_{5}$ und theilweise der gesammten $\mathrm{SO}_{3}$ über 68 Tage mit dazwischenliegenden Versuchstagen ausgedehnt wurden.

Es ergiebt sich aus diesen Versuchen, dass von dem

\begin{tabular}{|c|c|c|c|}
\hline ameiser & $\mathrm{NH}_{3}$ & 1,76 & pCt. \\
\hline essigsauren & - & 5,07 & - \\
\hline phosphorsauren & - & 29,98 & - \\
\hline sshwefelsauren & - & 35,86 & - \\
\hline salzsauren & - & 43,25 & - \\
\hline
\end{tabular}

des Ammoniakcomponenten durch den Harn zur Ausscheidung gelangten.

Dieser Ausscheidung des Ammoniakcomponenten gegenüber ergiebt die Untersuchung der Säurecomponenten, dass von der eingeführten Phosphorsäure 70,43 , von der eingeführten Schwefelsăure 56,39 pCt. im Harn wieder ausgeschieden sind. Es ist also in beiden Fällen weit weniger rom Ammoniakcomponenten zur Ausscheidung gekommen, als vom Säurecomponenten.

Bei dem Vergleich der Ausscheidungstage ergiebt sich aber, dass die Ausscheidung der Säuren viel schneller vor sich geht, als diejenige des Ammoniaks.

Die zweite Versuchsreihe wurde an einem Hunde von $18 \mathrm{~kg}$ Gewicht 
angestellt, bei welchem ebenfalls $\mathrm{N}, \mathrm{NH}_{3}$ und $\mathrm{P}_{2} \mathrm{O}_{5}$ untersucht wurden. Hier fübrten wir die Ammonsalze Anfangs per os, später subcutan ein.

Dabei ergab sich, dass von dem subcutan eingefübrten kohlensauren A m moniak 0,37 pCt. zur Ausscheidung kamen, ein Resultat, welches so gering ist, dass man dasselbe gegenüber 99,63 pCt., welche nicht wieder ausgeschieden wurden, als ein negatives betracbten kann. Dagegen wurden von dem subcutan eingefäbrten citronensauren Ammonium $23,88 \mathrm{pCt}$. und von dem subcutan eingeführten Ammonium phosphoricum 87,43 pCt. wieder durch den Harn ausgeschieden. Ueberraschender Weise fübrte die anfängliche Einführung von phosphorsaurem Ammoninm per os. zu einer Ausscheidung, welche die Einfuhr übertraf. Die Untersuchung der Phosphorsäureausscheidung ergab aber bei diesem Versuch, dass von dem subcutan eingeführten Säurecomponenten 52,86 pCt. zur Ausscheidung gelangten, während von dem eingeführten Ammoniakcomponenten $87,43 \mathrm{pCt}$. ausgeschieden wurden.

Etwas anders war das Ergebniss bei einem Phthisiker, bei welchem von dem eingeführten essigsauren, ameisensauren, phosphorsauren und sch w efelsauren Ammonsalz nur von dem letzteren 31,47 pCt. des Ammoniakcomponenten zur Ausscheidung gelangten, während sich von dem Schwefelsãurecomponenten nur 5,79 pCt. wiederfinden liessen.

Nach Einfuhr von 6,424 $\mathrm{g} \quad \mathrm{NH}_{3}$ and 13,46 $\mathrm{P}_{2} \mathrm{O}_{5}$ fand sich keine Ammoniakvermehrung, wohl aber 9,65 pCt. des Phosphorsäurecomponenten im Urin.

Ein weiterer eingehender Versuch wurde bei einem Hunde mit gleichzeitiger Bestimmung der Schwefel- und Phosphorsäureausscbeidung durch Harn und Kotb angestellt.

Derselbe schied in der Norm (11 Tage) pro Tag $5,46 \mathrm{~N}, 0,8084 \mathrm{NH}_{3}$, $0,6377 \mathrm{SO}_{3}$ und $0,688 \mathrm{P}_{2} \mathrm{O}_{5}$ durch den Harn, 0,2759 $\mathrm{SO}_{3}$ und $1,235 \mathrm{P}_{2} \mathrm{O}_{5}$ durch den Stuhl aus.

Vom 12. Untersuchungstage $a b$ erbielt er in 6 Tagen $45 \mathrm{~g} \mathrm{NH}_{4} \mathrm{SO}_{4}$ $=11,59 \mathrm{NH}_{3}$ und $27,274 \mathrm{SO}_{3}$. In dieser.Zeit, sowie in weiteren 7 Tagen schied derselbe $65,99 \mathrm{~N}, 27,783 \quad \mathrm{NH}_{3}, 17,375 \mathrm{SO}_{3}$ durch den Harn, $6,243 \mathrm{SO}_{3}$ und $28,816 \mathrm{P}_{2} \mathrm{O}_{5}$ durch den Koth aus. Es ergiebt sich daraus, dass $5,685 \mathrm{~g} \mathrm{NH}_{3}$ mehr ausgeschieden als eingenommen sind $=149$ pCt. der Einnahme. Von der Schwefelsāure wurden im Ganzen durch Harn und Stubl 23,6182 ausgeschieden. Da die Normalausscbeidung in dieser Zeit im Harn und Stubl $11,8755 \mathrm{~g}$ betragen bätte und 27,274 eingegeben sind, so bleibt ein Verlust von $15,53 \mathrm{~g} \mathrm{SO}_{3}$. Es sind somit nur 43,0 pCt. der eingeführten Schwefelsäure wieder zur Ausscheidung gelangt.

Allerdings wird gleichzeitig durch den Harn ond vor Allem durch den Koth wesentlich mehr Phosphorsäure ausgeschieden, als normal.

Während die Normalausscheidung dieser $\mathbf{1 , 9 2 3}$ pro die beträgt, steigt diese Zahl während der Einnahme von $\mathrm{SO}_{3}$ auf 2,99. Es hat somit die 
Zurückhaltung von $\mathrm{SO}_{3}$ gleichzeitig eine vermehrte Ausscheidung von $\mathrm{P}_{2} \mathrm{O}_{5}$ bewirkt.

Interessant ist auch, dass die Ausscheidung des gesammten $\mathbf{N}$ durch den Harn in dieser Periode beträchtlich geringer ist, als in der normalen Zeit. Da von diesem 34,6 pCt. auf $\mathrm{NH}_{3}$ entfallen, so muss, in Folge der Ueberschwemmung des Körpers mit schwefelsaurem Ammonium, die Bildung der normalen Harnstoffmenge eine wesentliche Beeinträchtigung erlitten haben.

Fassen wir das Resultat dieser Untersuchungen kurz zusammen! Es ergiebt sich zunächst, dass das an schwächere Säuren gebundene Ammoniak leichter im Körper verschwindet, als dasjenige festerer Bindung. So verschwindet das an Koblensäure gebundene $\mathrm{NH}_{3}$ fast völlig, wie das anch die Untersuchungen von $M \ddot{n} \mathbf{z}$ er und Neustatt aus der Klinik von v. Jacksch vor Kurzem gezeigt haben. Ameisensaures und essigsaures Ammonium lassen das $\mathrm{NH}_{3}$ theils völlig verschwinden, theils werden nur geringe Mengen desselben wieder ausgeschieden. Von phosphorsaurem Ammonium verschwindet das $\mathrm{NH}_{3}$ ebenfalls einmal im Körper, während in den meisten Versuchen von diesem, sowie von scbwefelsaurem und salzsaurem Ammoniumeinmehroderwenigergrosser Antheil des Ammoniakcomponenten im Harn zur Ausscheidung gelangt.

Aber diese Ausscheidung kann unmöglich in Bindung an den Säurecomponenten des eingeführten Salzes erfolgen.

Die Untersuchung der ausgeschiedenen Säurecomponenten lässt vielmehr nur den Scbluss zu, dass im Körper das betreffende A moniumsalz zu einem mehr oder weniger grossen Theil in seine Bestandtheile zerlegt wird. In einzelnen Fällen scheint die Phosphorsäure rasch eliminirt zu werden, während das Ammoniak in anderweitiger Bindung noch im Körper zurückbleibt; in anderen Fällen wird die eingeführte Säure zurückgebalten. Bezüglich des Chlorammoniums hat übrigens Feder eine ähnliche Zurückhaltung des Chlor im Körper gefunden.

Wenn nun dem Körper die Fähigkeit innewohnt, alle diese Ammonsalze zu zersetzen und je nach angenblicklichem Bedarf die eingeführten Quotienten der Säure auszuscheiden oder zurückzubalten, so dürfte Hallervorden's Anscbaung, dass das $\mathrm{NH}_{3}$ im Körperdie Function der Säureneutralisation zu üben hat, in diesereinseitigen Fassungnicht gültig sein. Denn da beispielsweise die Pbosphorsäure des phosphorsauren $\mathrm{NH}_{3}$, in einzelnen Fällen in grösserer Menge und viel rascher aus dem Körper ausgeschieden wird, als das Ammoniak, so kann das Resultat des Prozesses nur in einer gesteigerten Na-Ausfuhr aus dem Körper bestehen.

Bei dem zuletzt angeführten Stoffwechselversuch beim Hunde bleiben aber 60 pCt. der eingeführten Schwefelsäure im Körper, während die Ammoniakausfuhr durch den Harn die Einfuhr um 50 pCt. übersteigt.

Wo bleibt da Ballervorden's Bindung der Säuren durch $\mathrm{NB}_{8}$ ? 
Viel verwickelter gestaltet sich nach diesen Versuchen die Ausscheidung der Ammoniaksalze. Ausserordentlich rasch müssen sich die Wegedes $\mathrm{NH}_{3}$ und der zugehörigen Säure im Körpertrennen.

Nach den Untersucbungen von $\vee$. Scbrōder dürfen wir wobl mit Recht annehmen, dass diese Trennung zum Theil in der Leber vor sich gebt, zumal sicb in dieser für die Schwefelsäure einige Gelegenheit zu anderweitiger Bindung findet. Ich erinnere nur an diejenige mit den aromatischen, zum Theil hochgradig giftigen Verbindungen, weiterhin an das schwefelsăurehaltige Taurin, den Componenten der in der Leber gebildeten Taurocholsäure.

Uebrigens dürfte auch ein kleiner Theil des Ammoniaks in der Leber zur Bildung von Glykocoll Verwendung finden.

Die freiwerdende Phosphorsäure könnte eben so gut zum Aufbau des Lecithins dienen, wie sie mit einer anderen Base weiter im Blute kreisen kann, um hier in mannichfache Wechselwirkungen zu treten oder auch ausgeschieden zu werden.

Denkbar wäre es auch, dass das phosphorsaure Natron sich dem in den Geweben bei dem Zerfall von Eiweiss freiwerdenden $\mathrm{NH}_{3}$ unter Freigabe des $\mathrm{Na}$ an Koblensăure zur Verfügung stellt, um nach Passage der Leber den gleichen Kreislauf von Neuem zu beginnen. Naturgemäss würde das nicht hindern, dass die Phosphorsäure theilweise durch Chlor aus ihrer Verbindung mit $\mathrm{NH}_{3}$ ansgetrieben wird. Das Chlorammonium aber, welches der Umwandlung in Harnstoff grossen Widerstand entgegen zu setzen scheint, würde dadurch schon lănger im Körper kreisen. Dass aber auch dieses Salz in seine Componenten zerlegt und diese nicht gleichmässig und gleicbzeitig ausgeschieden werden, lebren $\mathrm{F}$ eder's Untersuchungen. Körper?

In welcher Form kreist nun die grōssere Menge von Ammoniak im

In dieser Beziehung sind vielleicht die toxischen Erscheinungen bei der Einfuhr von Ammoninmsalzen von Interesse. Alle Verbindungen des Ammonium, insbesondere die lockeren Bindungen entfalten toxische Wirkungen. Dieselben treten vor Allem nach mebrfach wiederholten subcutanen Injectionen auf. Am stärksten sind dieselben bei kohlensaurem Ammonium, bestehend in Erbrechen, Speichelfluss, grosser Hinfälligkeit, Tremor u.s. w.

Weiterhin war die subcutane Einführung von grösseren Mengen koblensauren Ammoniaks von einer Nekrose der Haut an der Injectionsstelle gefolgt. Bei solchen Wirkungen des kohlensauren $\mathrm{NH}_{3}$ ist kaum zu denken, dass dasselbe als wesentlichste Vorstufe des Harnstoffs im Körper kreist.

Aber diese toxischen Eigenschaften der Ammoniumsalze lassen doch daran denken, dass denselben in einzelnen Krankheitszuständen eine Bedeutung zukommt. Ob dabei die Anschaung von Frerichs in der Weise modificirt wird, dass nicht der Zerfall des Harnstoffs in Ammoniumverbindungen, sondern die mangelnde Umwandlung dieser in Harnstoff zu toxischen Erscheinungen führt, muss die Zukunft lehren. 


\section{8}

In dieser Hinsicht erscheint es mir von Wichtigkeit, dass eine gewisse Ueberschwemmung des: Körpers mit Ammoniumverbindungen selbst die Bildung der mormalen Harnst offmenge hemmt.

Da diese Bildung nach v. Schröder's Untersuchungen in der Leber statt bat, so ergiebt sich aus obigen Befunden die weitere Frage, ob nicht auch in Krankbeiten diese Function der Leber leidet.

Es wird naturgemäss noch vieler Untersuchungen bedürfen, ehe diese Fragen sich befriedigend beantworten lassen. Aber Hallervorden wird aus dem Mitgetheilten ersehen, dass die Frage der $\mathrm{NH}_{3}$-Ausscheidung weit entfernt von dem Abschlusse ist, welchen er anzunehmen geneigt ist.

Ich erkenne aber gern die grossen Verdienste an, welche sich die Schüler Naungn's und Scbmiedeberg's um die Frage der $\mathrm{NH}_{3}$-Ausscheidung erworben haben.

Aber neue Befunde stellen neue Fragen, wenn dabei auch liebgewordene Anschauungen fallen müssen.

Eine voreilige Zufriedenheit muss zum Stillstand führen. 